\title{
Alterstice
}

Revue internationale de la recherche interculturelle

International Journal of Intercultural Research

Revista International de la Investigacion Intercultural

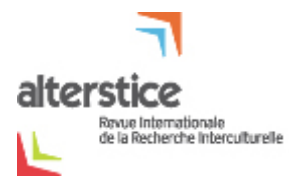

\section{La culture en tant que facteur du développement cognitif}

\section{Jean Retschitzki}

Volume 1, numéro 1, 2011

URI : https://id.erudit.org/iderudit/1077593ar

DOI : https://doi.org/10.7202/1077593ar

Aller au sommaire du numéro

\section{Éditeur(s)}

Alterstice

ISSN

1923-919X (numérique)

Découvrir la revue

Citer cet article

Retschitzki, J. (2011). La culture en tant que facteur du développement cognitif. Alterstice, 1(1), 81-93. https://doi.org/10.7202/1077593ar

\section{Résumé de l'article}

Cet article discute du rôle de la culture en tant que facteur du développement cognitif, à partir d'un certain nombre de travaux en psychologie interculturelle du développement cognitif. L'évolution des recherches dans ce domaine, tant sur le plan méthodologique que dans la manière d'aborder cette problématique à la fin du vingtième siècle, est présentée et commentée.

Après un tour d'horizon des principaux travaux empiriques ayant cherché à déterminer dans quelle mesure le développement cognitif est universel ou relatif aux groupes culturels, nous exposons les évolutions récentes dans le domaine de la psychologie interculturelle du développement cognitif, sous l'influence notamment du courant socioculturel issu principalement des travaux de Vygotsky. Nous présentons les principales caractéristiques des travaux qui se réclament de la psychologie culturelle et nous en évaluons la portée. 


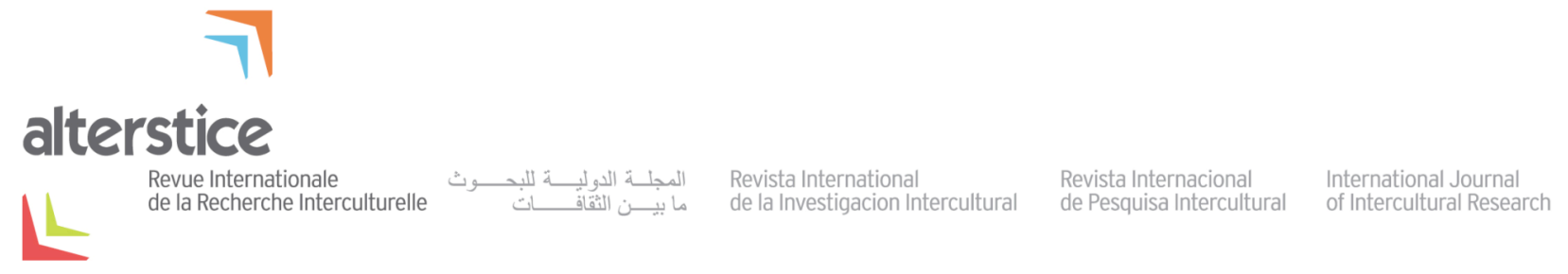

ARTICLE HORS THÈME

\section{La culture en tant que facteur du développement cognitif}

Jean Retschitzki ${ }^{1}$

\section{Résumé}

Cet article discute du rôle de la culture en tant que facteur du développement cognitif, à partir d'un certain nombre de travaux en psychologie interculturelle du développement cognitif. L'évolution des recherches dans ce domaine, tant sur le plan méthodologique que dans la manière d'aborder cette problématique à la fin du vingtième siècle, est présentée et commentée.

Après un tour d'horizon des principaux travaux empiriques ayant cherché à déterminer dans quelle mesure le développement cognitif est universel ou relatif aux groupes culturels, nous exposons les évolutions récentes dans le domaine de la psychologie interculturelle du développement cognitif, sous l'influence notamment du courant socioculturel issu principalement des travaux de Vygotsky. Nous présentons les principales caractéristiques des travaux qui se réclament de la psychologie culturelle et nous en évaluons la portée.

\section{Rattachement de l'auteur \\ ${ }^{1}$ Département de Psychologie, Université de Fribourg, Fribourg, Suisse}

\section{Correspondance}

jean.retschitzki@unifr.ch

\section{Mots clés}

développement cognitif; psychologie culturelle; rôle de la culture; comparaison; développement; facteur du développement; universalité

\section{Pour citer cet article :}

Retschitzki, J. (2011). La culture en tant que facteur du développement cognitif. Alterstice, 1(1), 81-94. 


\section{Introduction}

Tout être humain commence sa vie en relation étroite avec son milieu social, dont il est totalement dépendant; son développement tant affectif que social ou cognitif est évidemment influencé par ce lien initial très fort, notamment au cours des phases initiales.

En plus des contraintes biologiques et des caractéristiques du milieu physique, la culture qui caractérise ce milieu social avec ses différentes facettes (connaissances, croyances, morale, droit, coutumes, valeurs, etc.) joue un rôle dans la manière dont l'individu se développe. Par exemple, cette influence peut se manifester en autorisant ou en favorisant certaines potentialités, tout en cherchant au contraire à éviter, prévenir, voire interdire la pratique d'autres conduites.

On peut raisonnablement faire l'hypothèse que le développement cognitif est particulièrement sensible aux effets des modes de vie, des circonstances particulières du milieu physique comme du milieu social.

Comme le souligne Troadec dans l'introduction à son ouvrage consacré à la psychologie culturelle, « il est rare que la culture, bien qu'essentielle, soit au centre de la démarche » dans les travaux de recherche sur le développement de la cognition (Troadec, 2007, p. 9).

Le projet même de la psychologie interculturelle, au moins dans ses aspects comparatifs, sous-entend que la culture et les aspects sociaux jouent un rôle significatif dans les comportements. Les recherches visent donc souvent à pondérer l'importance relative des différents facteurs et à décrire les processus sous-jacents.

Dans leur ouvrage d'introduction à la psychologie interculturelle, Segall, Dasen, Berry et Poortinga (1999, p. 26) ont présenté un "cadre conceptuel pour la psychologie interculturelle» qui met en évidence différents aspects contextuels et processus susceptibles d'avoir une influence culturelle sur le développement individuel: contexte socio-politique, adaptation culturelle, transmission culturelle, acculturation.

Plus récemment Dasen $(2003 ; 2004)$ a proposé une intégration de ce cadre conceptuel en combinant cette vision avec la niche développementale de Super et Harkness (1986) et la théorie des systèmes écologiques de Bronfenbrenner (1979). Cette nouvelle présentation insiste sur les interactions multiples et les rétroactions.

L'objectif principal de cet article est de discuter du rôle de la culture en tant que facteur du développement cognitif, à partir d'un certain nombre de travaux en psychologie interculturelle du développement cognitif. Il s'agit également de montrer l'évolution des recherches tant sur le plan méthodologique que dans la manière d'aborder cette problématique au cours du dernier tiers du vingtième siècle.

Après une présentation des principaux travaux empiriques ayant cherché à déterminer dans quelle mesure le développement cognitif est universel ou relatif aux groupes culturels, nous analyserons les évolutions récentes dans le domaine de la psychologie interculturelle du développement cognitif, sous l'influence du courant socioculturel issu des travaux de Vygotsky. Nous chercherons en particulier à mettre en évidence les principales caractéristiques des travaux qui se réclament de la psychologie culturelle, et à en évaluer la portée.

\section{Diverses orientations en psychologie interculturelle}

Les auteurs sont loin d'être unanimes quant à leurs présupposés théoriques. C'est ainsi qu'on recense classiquement trois orientations principales vis-à-vis de la problématique interculturelle : absolutiste, universaliste, et relativiste.

Pour les tenants de l'orientation absolutiste, les phénomènes psychologiques sont considérés comme étant les mêmes dans toutes les cultures, si bien que les comparaisons ne sont pas considérées comme posant des problèmes essentiels. 
Les partisans de l'orientation universaliste considèrent que les processus psychologiques fondamentaux ont probablement des caractéristiques communes à la vie humaine en tout lieu, mais que leurs manifestations sont probablement influencées par la culture. Les recherches comparatives peuvent donc être entreprises, mais après une étude minutieuse des conditions de vie de chaque groupe culturel et en prenant soin d'adapter les instruments utilisés aux caractéristiques du (ou des) groupe(s) étudié(s), ce qui n'est pas toujours simple.

Enfin les auteurs qui adoptent une orientation relativiste pensent que l'explication des variations psychologiques entre les peuples du monde doit être cherchée en termes de variation culturelle avec peu de recours à d'autres facteurs. Dans une telle perspective, les études comparatives sont évitées, parce qu'elles sont considérées si problématiques et si ethnocentriques que toute comparaison valable est rendue impossible. Bril et Lehalle (1988, p. 92-93) résument cette position de la manière suivante :

Le point commun des auteurs qui se situent dans cette perspective est de considérer que toute activité intellectuelle est " contextualisée » et donc les réponses des sujets dans les tâches cognitives doivent s'analyser selon les attitudes et les représentations spécifiques à leur culture [...] Les recherches interculturelles sur la cognition devraient partir de l'analyse des pratiques culturelles régionales (activités professionnelles, jeux traditionnels, etc.).

Le lecteur souhaitant approfondir sa connaissance des trois approches et de leurs implications pour la recherche interculturelle peut se référer à la discussion présentée dans l'ouvrage de Berry, Poortinga, Segall et Dasen (2002, p. 324-328).

Cole, I'un des avocats de l'approche relativiste, a schématisé et comparé quatre grandes conceptions théoriques en psychologie du développement et il met en évidence le poids relatif des facteurs du développement dans ces systèmes théoriques. Le point commun entre les trois premières visions est que le développement est vu comme résultant d'une interaction entre deux forces juxtaposées (biologie, milieu); les différences entre ces conceptions proviennent du poids relatif des contraintes biologiques ou de l'expérience et des actions de l'enfant sur le milieu.

Une première approche théorique, appelée "biologique-maturationnelle », dont Gesell serait l'auteur le plus typique selon Cole, met l'accent avant tout sur les facteurs biologiques et la maturation du système nerveux; le rôle de l'environnement y est réduit à sa plus simple expression. À l'inverse, la deuxième conception, que Cole attribue à Skinner, étiquetée "apprentissage-milieu», met l'accent sur l'influence dominante du milieu dans le développement, les aspects biologiques ne jouant qu'un rôle mineur. La troisième conception, la position " interactionniste » de Piaget, est intermédiaire dans le sens où les influences des aspects biologiques et du milieu sont de poids comparable, l'important étant, selon Piaget, le fait que l'organisme se développe en cherchant activement à s'adapter à son milieu.

La spécificité de la quatrième conception théorique, l'approche "culturelle ", dont se réclame Cole, réside dans l'inclusion de la culture comme constituant inséparable du développement.

Selon cette perspective " contexte culturel ", les deux facteurs appelés biologie et environnement - ou l'individu et la société - dans les approches décrites auparavant n'interagissent pas directement. Leur interaction est médiatisée à travers un troisième facteur, la culture (Cole, 1992, p. 735 - notre traduction) ${ }^{1}$.

Les principes de base de la psychologie culturelle-historique de Cole sont développés dans plusieurs publications (Cole, 1992; 1995; 1996). En ce qui concerne le développement historique, Cole insiste sur le fait qu'en plus de l'utilisation et de la fabrication d'outils, les êtres humains organisent la redécouverte des outils déjà créés dans chaque génération successive. La culture peut donc être comprise comme la totalité des artefacts accumulés par le groupe social au cours de son expérience historique. Sur le plan des activités pratiques du chercheur, Cole est d'avis que l'analyse des fonctions psychologiques doit être fondée sur les activités quotidiennes. Dans la mesure où les premiers travaux de cet auteur peuvent être considérés comme appartenant à la psychologie interculturelle comparative "classique ", il peut être intéressant de considérer les motivations qui l'ont conduit à passer de cette

\footnotetext{
${ }^{1}$ According to this cultural context view, the two factors labeled biology and the environment or the individual and the society in the previously described approaches do not interact directly. Rather, their interaction is mediated through a third factor, culture.
} 
première orientation à sa conception actuelle; un article publié dans la Revue Suisse de Psychologie explicite les motivations de cette évolution (Cole, 1995).

Ces présentations très contrastées ne reflètent évidemment que de manière approximative la réalité des travaux empiriques. Nombre d'entre eux pourraient être classés dans des catégories intermédiaires. Dans ceux auxquels nous avons collaboré ou que nous avons menés dans le domaine interculturel, la perspective a été clairement universaliste, influencée par la pensée piagétienne. Mais cette orientation initiale a été tempérée par le fait que l'anticipation des critiques relativistes a amené nombre de chercheurs à prendre des précautions dans l'adaptation des instruments, voire à adopter une approche " émique » (souci de se représenter les choses du point de vue des populations concernées) plutôt qu'une approche "étique», dans laquelle on peut voir l'influence directe ou indirecte de l'approche relativiste.

Appliquée aux recherches interculturelles, l'approche émique consiste alors à partir de l'observation des comportements et représentations locales en s'interdisant a priori (mais pas nécessairement a posteriori) l'application dans telle ou telle culture des grilles d'observation ou des situations expérimentales utilisées dans d'autres. De son côté, l'approche étique cherche à élaborer des concepts généraux qui transcendent les réalisations culturelles particulières. Par exemple, en ce qui concerne les opérations intellectuelles, les études émiques s'appuieront en premier lieu sur les pratiques intellectuelles en vigueur dans la culture considérée [...]. (Bril et Lehalle, 1988, p. 14-15)

\section{Recherches sur l'universalité du développement cognitif}

Pour illustrer notre propos, il n'est pas superflu de considérer quelques résultats de recherches portant sur les différentes périodes du développement intellectuel envisagé en référence aux périodes du développement décrites par Piaget.

\section{Période sensori-motrice (I)}

Le développent de l'intelligence sensori-motrice a donné lieu à relativement peu de travaux empiriques, sans doute en raison des difficultés pratiques d'une telle entreprise. Notre étude en Côte d'Ivoire (Dasen, Inhelder, Lavallée et Retschitzki, 1978) a permis de mettre en évidence la présence de conduites qualitativement identiques à celles observées par Piaget sur ses propres enfants et codifiées ensuite dans l'échelle de développement de Casati et Lézine. De même en ce qui concerne le rythme de développement, le résultat le plus frappant a été la similitude entre les résultats des bébés baoulés avec les normes françaises. Les seules différences ont consisté dans la précocité des petits baoulés pour certains éléments qu'on pouvait considérer comme proches des conduites que ces bébés pouvaient observer ou pratiquer eux-mêmes dans leur environnement (moins pauvre en objets que ce que certaines descriptions pouvaient laisser supposer).

Parmi les facteurs susceptibles d'expliquer ces données on peut mentionner la précocité relative du développement moteur, la possibilité de manipuler très tôt nombre d'objets présents dans le milieu, la stimulation (sensorielle et relationnelle) des bébés du fait de leurs conditions de vie particulières et la relation très étroite entre la mère et le bébé durant la première année de la vie. Ce qui a amené Langley-Danysz (1979) à évoquer a contrario un « handicap du berceau » pour décrire les conditions de vie d'un bébé élevé à l'occidentale.

\section{Opérations concrètes (II)}

C'est la période pour laquelle les études sont les plus nombreuses. Il n'est pas question ici d'en faire une recension exhaustive. Tout au plus pourrons-nous évoquer les grandes tendances des études comparatives.

Là encore l'impression dominante est la similitude qualitative des résultats : on retrouve généralement les mêmes conduites, les mêmes stades et dans le même ordre. Les différences observées portent sur les aspects quantitatifs; en particulier la chronologie peut être modifiée par les influences éducatives ou culturelles qui s'exercent sur les individus.

La conservation des quantités semble être un universel; toutefois Dasen a trouvé des courbes " asymptotiques » dans certains groupes, indiquant que seule une partie des sujets atteignent le stade ultime. Cette constatation l'a 
conduit à faire l'hypothèse que cette épreuve mettait parfois en évidence une performance plutôt qu'une compétence; des expériences ultérieures utilisant des techniques d'apprentissage ont en effet permis de déclencher assez rapidement cette acquisition (Dasen, 1975).

Ces résultats ont été corroborés par des études montrant le rôle de l'acculturation. En comparant des groupes d'Aborigènes australiens dont la durée de contact avec la culture dominante anglophone variait, il a pu montrer que les résultats étaient toujours en faveur de l'échantillon ayant le plus de contact. Utilisant des épreuves de différents domaines cognitifs, il a même pu montrer que l'influence de l'acculturation est plus importante pour les concepts qui sont moins pertinents dans la culture, comme les conservations.

Un autre aspect des travaux de Dasen a consisté à étendre les idées du modèle éco-culturel de Berry (1976), conçu initialement pour étudier l'influence des facteurs culturels sur la dépendance-indépendance à l'égard du champ, au domaine du développement intellectuel. Dasen a fait l'hypothèse que les notions spatiales se développeraient plus rapidement dans les populations nomades que dans les groupes sédentaires et que les notions de conservation se développeraient plus vite dans les populations sédentaires que dans les groupes nomades. Dans les grandes lignes, les résultats ont été conformes à l'hypothèse pour le domaine spatial, mais un peu moins nets pour les conservations, seuls les sujets plus âgés (12 à 14 ans) présentant la configuration de résultats attendue.

La tendance indiquée par ces résultats est que le développement est d'autant plus précoce que le domaine concerné est fortement valorisé par le groupe culturel.

On peut interpréter ces résultats en référence aux influences volontaires, organisées, voire formelles exercées sur les enfants sous forme d'encouragements, d'enseignement ou même d'un ensemble de récompenses et de punitions, mais aussi aux influences involontaires, informelles provenant des situations ou notions vécues, utilisées, exercées dans les activités quotidiennes ou véhiculées par le langage (richesse du vocabulaire, p. ex.)².

Par exemple, un enfant africain élevé dans un milieu traditionnel, comme c'est le cas des enfants interrogés par Dasen, est très tôt confronté à des notions numériques à travers les échanges qui se déroulent sur les marchés, présents dans tout village ou tout quartier d'une ville, et où le bébé accompagne le plus souvent sa mère dès sa plus tendre enfance.

\section{Opérations formelles (III)}

La littérature est assez pauvre concernant la période des opérations formelles. À ce niveau, il est pratiquement impensable de simplement répliquer les études menées à Genève avec un matériel et des problèmes qui se justifiaient dans le contexte d'une société où la formation scientifique a une place éminente. Ces épreuves (induction expérimentale, combinatoire, etc.) font partie des contenus enseignés dans les écoles secondaires; elles se prêtent mal à des études comparatives dans d'autres cultures; elles sont difficiles à transposer, à adapter.

Du fait qu'on ne disposait que de peu de résultats probants quant à la présence d'opérations formelles dans des groupes ethniques non occidentaux, certains auteurs ont émis l'hypothèse que les opérations formelles pourraient avoir des pré-requis liés à un certain type de culture. Ainsi Scribner (1969) a défendu l'idée que l'alphabétisation pourrait être une condition nécessaire pour parvenir au stade des opérations formelles.

Jahoda (1980) a pris une position très différente en soulignant que de nombreux individus des sociétés traditionnelles peuvent fonctionner à un niveau formel, mais dans des secteurs qui leur sont familiers.

En allant plus loin, on peut remarquer que la forme particulière de raisonnement à l'œuvre dans ces épreuves, le raisonnement scientifique, n'est certainement pas ce qui est valorisé dans toutes les cultures. C'est également

\footnotetext{
${ }^{2}$ La littérature sur les savoirs quotidiens oppose les connaissances formelles acquises à travers des systèmes organisés par la société, principalement l'école, aux connaissances informelles acquises dans la vie quotidienne, en dehors des institutions.
} 
I'opinion de Greenfield (1976), qui préconise de suivre l'exemple de Piaget lui-même : déterminer d'abord quel est le stade final, puis en étudier l'ontogenèse, mais ceci selon les valorisations particulières de chaque société.

Les recherches menées à Recife par Nunes, Schliemann et Carraher (1993) ont montré la capacité d'adultes faiblement scolarisés (charpentiers, agriculteurs et pêcheurs) à utiliser et même à transférer des raisonnements combinatoires et de proportionnalité utilisés dans leur pratique professionnelle quotidienne.

Il est intéressant de mentionner la contribution originale de Tapé (1994), chercheur ivoirien, qui a utilisé une technique originale pour étudier dans sa propre ethnie (bété) le raisonnement de sujets adultes non scolarisés en ayant recours à trois épreuves piagétiennes (la flexibilité des tiges, le pendule, les permutations). Il indique que les adultes approchent les problèmes avec une vision globale, regroupent des propriétés en blocs d'information, et ordonnent les facteurs. Par exemple, dans l'expérience du pendule, ils considèrent que le facteur principal est l'élan, puisque sans lui il n'y aurait aucun mouvement. Ils tiennent aussi compte de l'expérience de la vie quotidienne : par exemple, dans la flexibilité des tiges, ils considèrent que l'expérience n'est pas valable telle qu'elle est présentée, mais qu'il faut mettre la flexibilité des matériaux à l'épreuve du temps, comme pour les tiges de bois utilisées pour la construction de pièges. Pour les permutations, les adultes illettrés rencontrent des difficultés à partir de 4-5 objets, car en l'absence d'écriture, ils ne gardent pas la trace des permutations déjà effectuées.

Tapé conclut qu'il y a deux styles de pensée qui se départagent à l'adolescence, et qui correspondent à deux modèles interprétatifs de la nature. Au style expérimental et analytique correspond la logique formelle, la question "comment? " et la détermination de la causalité; au style "expériencé ", qui procède par représentation symbolique et figurale, correspond la logique pragmatique ou réalisatrice, la question " pourquoi? » et la recherche d'une finalité. Selon Tapé, ce second style, correspondant à la philosophie bantoue, est favorisé par l'éducation informelle, et il se marque dans la façon dont les adultes non scolarisés abordent et résolvent les tâches proposées.

L'une des réserves qu'il convient de signaler à propos des travaux de Tapé concerne la méthode d'investigation : l'auteur a interrogé ses sujets selon une méthode d'entretien de groupe ou "entrevue interactive », selon lui culturellement plus appropriée que les examens individuels, mais qui rend toute comparaison avec les données d'origine très délicate.

Au terme de ce bref survol de quelques travaux comparatifs, on peut conclure avec Dasen $(1997$, p. 152) que

certains aspects du développement cognitif semblent universels: les structures cognitives sensori-motrices et des opérations concrètes, au moins en ce qui concerne la compétence, et les mécanismes et les étapes de leur construction. Mais les rythmes de développement selon les domaines notionnels, l'actualisation de la compétence en performance, les contenus auxquels s'appliquent les structures, et les contextes dans lesquels elles sont mises en œuvre dépendent largement des valorisations culturelles.

Ces valorisations ne nécessitent aucune prise de conscience ou volonté délibérée, même si elles ne les excluent pas; elles trouvent tout naturellement leur traduction dans l'importance accordée à telle ou telle connaissance explicite ou implicite, à tel ou tel savoir-faire, dans la richesse du vocabulaire du ou des domaines valorisés, etc.

\section{Évolutions récentes : l'impact de la psychologie culturelle}

Au cours des dernières décennies, l'expression «psychologie culturelle » a connu un succès certain dans la littérature. À y regarder de plus près, on est toutefois amené à considérer qu'il n'y a pas d'unité de doctrine entre tous les auteurs qui se réclament de ce courant; la diversité est même très importante.

La psychologie culturelle a des origines lointaines, puisque certains auteurs comme Cole (1995) en voient les prémisses dans les travaux de Wundt consacré à la "Völkerpsychologie " (Wundt, 1912). Plus récemment, cette perspective a été adoptée par plusieurs groupes qui partagent une vision relativiste et mettent l'accent sur des aspects plus subjectifs. Shweder affirme par exemple que «la psychologie culturelle est l'étude des mondes intentionnels " (Shweder, 1990, p. 3). La psychologie culturelle postule la cohérence d'une culture considérée comme un système. 
Parmi les courants qu'on peut rattacher à la psychologie culturelle figure la théorie de l'action (Boesch, 1995). Pour ce dernier le développement de l'individu est à comprendre comme le résultat de transactions avec le milieu physique et surtout le milieu social. Nous sommes non seulement façonnés par notre environnement, mais nous contribuons à le former; à travers nos actions, nous pouvons modifier le cours des événements.

Deux auteurs ont eu à notre avis une influence déterminante dans l'émergence de la psychologie culturelle : Vygotsky et Bruner. L'influence de Vygotsky est particulièrement manifeste dans les travaux de Cole et du courant socio-culturel. Dans cette approche, l'accent est mis davantage sur le langage et les aspects sociaux comme facteurs du développement; c'est la culture qui fournit les outils (matériels ou symboliques) permettant le développement.

Mais l'influence de Bruner a sans doute été déterminante dans le développement de la psychologie culturelle, notamment aux États-Unis. Après avoir été l'un des acteurs de la révolution cognitive (Bruner, Goodnow et Austin, 1956), Bruner a inspiré nombre de chercheurs importants dans le domaine (Cole et Greenfield, notamment). II a développé ses conceptions dans des ouvrages plus récents (Bruner, 1990; 1991; 1996). Ce que Bruner reproche surtout au courant cognitiviste est d'avoir mis de côté la "construction de la signification » au profit du "traitement de l'information». I propose au contraire de prendre en compte la "psychologie populaire », façonnée par la culture, "grâce à laquelle les gens organisent la vision qu'ils ont d'eux-mêmes, des autres, et du monde dans lequel ils vivent ». Selon lui, la psychologie populaire "n'est pas tant un ensemble de propositions logiques qu'une pratique du récit et du conte. Elle puise pour cela dans une inépuisable réserve de culture narrative: histoires, mythes, genres littéraires. " (Bruner, 1991, p. 146). Bruner ajoute que l'apprentissage et la pensée sont toujours situés dans un milieu culturel et toujours dépendants de l'utilisation des ressources culturelles (Bruner, 1996).

On retrouve dans ces citations certaines des idées qui ont permis l'émergence de nouvelles approches en psychologie interculturelle. Bien que sa base empirique soit relativement limitée, notamment en raison de la brièveté de la carrière de l'auteur en psychologie, la théorie de Vygotsky a eu une forte influence sur les chercheurs, tout comme les idées de Bruner. L'étude des «savoirs quotidiens » (everyday cognition) en est le prolongement, tout comme les conceptions de la "cognition située » (Brown, Collins et Duguid, 1989), de la " cognition partagée socialement » (Resnick, Levine et Teasley, 1991) et de la " cognition distribuée » (Salomon, 1993).

\section{Savoirs quotidiens}

Les travaux sur les savoirs quotidiens ont été l'occasion d'une collaboration souvent fructueuse entre psychologues et ethnologues ou anthropologues (Greenfield et Lave, 1978; Wassmann et Dasen, 1994a et 1994b). Pour optimiser la validité culturelle des recherches, les chercheurs ont tenté de mieux tenir compte des véritables savoirs des gens ordinaires (just plain folks) plutôt que de leurs performances à des épreuves psychologiques. Cette approche les a conduit à sortir du laboratoire pour s'impliquer dans des situations ordinaires et observer les pratiques des gens. Les conduites cognitives sont alors étudiées dans une situation naturelle, avec une combinaison de méthodes expérimentales et d'observation. De nombreuses études ont été menées à propos de domaines tels que le dénombrement (Wassmann et Dasen, 1994b), I'arithmétique (Nunes, Schliemann et Carraher, 1993; Lave, 1988), la classification (Wassmann et Dasen, 1994a), le tissage (Tanon, 1994; Greenfield, 2004).

Dans ce dernier ouvrage, richement illustré, Greenfield tire parti d'observations effectuées durant plus de trois décennies dans un village du Chiapas au Mexique. Elle montre non seulement les processus à l'œuvre dans la transmission des techniques artisanales, mais également leur évolution au cours de la période récente, ce qui lui permet aussi de mesurer l'impact de la mondialisation. Ces observations lui permettent en outre de décrire les relations mère-fille d'une manière plus personnelle.

Notons qu'on peut trouver une bonne introduction en français à la thématique des savoirs quotidiens dans les chapitres de Dasen et Bossel-Lagos (1989) et de Dasen (2004). 


\section{Cognition située}

En rupture avec le présupposé selon lequel la connaissance conceptuelle peut être abstraite des situations dans lesquelles elle est apprise et utilisée, présupposé sous-jacent à de nombreuses pratiques d'enseignement, Brown, Collins et leurs collègues ont proposé l'apprentissage cognitif (cognitive apprenticeship), une nouvelle manière de concevoir l'enseignement qui fait honneur à la nature située de la connaissance. Ces méthodes s'efforcent d'enculturer ${ }^{3}$ les élèves dans des pratiques authentiques à travers une activité et une interaction sociale d'une manière similaire à celles qui prévalent dans l'apprentissage des savoir-faire artisanaux (Brown, Collins et Duguid, 1989; Collins, Brown et Newman, 1989). Ce qui fait l'originalité de ces propositions est qu'on s'inspire des méthodes de transmission qui ont prévalu bien avant que les écoles ne voient le jour, pour transformer les pratiques d'enseignement dans les écoles d'aujourd'hui, y compris en tirant parti des possibilités offertes par les nouvelles technologies. C'est d'autant plus à souligner que des auteurs comme Collins et Brown ont fortement contribué dans les années 1960-1970 au développement de modèles informatiques, dont certains ont connu un succès notable dans des domaines tels que la mémoire (Collins et Quillian, 1969; Collins et Loftus, 1975) ou les opérations arithmétiques (Brown et Burton, 1978). Les domaines d'application de ces nouvelles méthodes d'enseignement vont de la résolution de problèmes en mathématiques à la rédaction de textes, en passant par l'enseignement réciproque de la lecture.

D'une manière similaire, Rogoff (1990) décrit de nombreuses situations quotidiennes (aux États-Unis et au Guatemala principalement, mais aussi en Asie, en Afrique, etc.) où intervient la participation guidée à des activités sociales avec des compagnons qui soutiennent et étendent la compréhension des savoir-faire par l'utilisation des outils de la culture. Elle montre l'universalité de la participation guidée tout en soulignant des différences culturelles relatives aux buts du développement (ce qui doit être appris) et aux moyens (possibilité d'observer et de participer aux activités). Dans un livre récent, Rogoff insiste sur la nature culturelle du développement, se basant à nouveau sur des observations effectuées dans de nombreuses cultures, aussi bien en Océanie qu'en Afrique, en Amérique latine qu'aux États-Unis (Rogoff, 2003).

La position des auteurs se situant dans le courant de la cognition située ne pouvait pas laisser indifférents les tenants d'une approche cognitiviste plus traditionnelle : c'est ainsi qu'Anderson et ses collègues ont souligné en quoi ils ne pouvaient se rallier à cette vision (Anderson, Reder et Simon, 1996). Ils citent quatre points qui leur semblent poser problème.

En premier lieu, à propos de la nature située de la connaissance, s'ils acceptent que certaines connaissances acquises dans des situations quotidiennes ne puissent pas être généralisées dans un contexte scolaire, ils affirment que cela ne suffit pas à démontrer que les connaissances scolaires ne peuvent pas être utilisées hors de l'école. Deuxièmement, les auteurs contestent que le transfert ne soit pas possible entre tâches. Ils affirment au contraire que de nombreuses données attestent que toutes les situations possibles existent : transfert important, peu de transfert, pas de transfert ou même transfert négatif.

Le troisième point sur lequel Anderson et ses collègues formulent une objection concerne l'affirmation du peu d'utilité de l'entraînement abstrait : selon eux l'enseignement abstrait peut être très puissant s'il est accompagné d'exemples concrets.

La quatrième objection d'Anderson, Reder et Simon réside dans le fait qu'ils ne pensent pas qu'il soit judicieux d'enseigner exclusivement dans des situations sociales complexes, mais qu'il convient de recourir aussi à des périodes d'entraînement individuel.

\footnotetext{
${ }^{3}$ Troadec propose la définition suivante pour le terme d'enculturation: "Procesus d'acquisition d'une culture ». Les auteurs anglophones distinguent une enculturation formelle (socialization), telle que celle du milieu scolaire, et une enculturation informelle (enculturation), telle que celle qui résulte de ce que l'on apprend au hasard dans la rue. (Troadec, 2007, p. 75)
} 
Un certain rapprochement des points de vue a été publié par la suite dans un article signé des mêmes auteurs conjointement avec Greeno (Anderson, Greeno, Reder, et Simon, 2000). Ils parviennent à des conclusions plus nuancées et s'accordent pour juger que de nouvelles recherches sont nécessaires pour améliorer les pratiques scolaires.

\section{Publications récentes}

Le courant de la psychologie culturelle est toujours très actif, comme l'attestent les nombreux livres publiés récemment par des spécialistes de la psychologie sociale. C'est ainsi que Ratner a publié au cours des dernières années deux ouvrages traitant de la psychologie culturelle (Ratner, 2002 et 2006). II se réfère explicitement à Vygotsky, Luria et Leontiev et souligne combien les processus sociaux sont à l'origine de la vie psychique, contribuent à la modeler et s'y reflètent. L'analyse culturelle lui semble nécessaire pour arriver à une véritable compréhension des réalités psychologiques, et elle peut fournir des repères pour améliorer la vie des êtres humains. Dans son ouvrage le plus récent, Ratner (2006) tente de démontrer l'impact de la culture dans la stimulation et la structuration de domaines aussi variés que les émotions, la personnalité, la perception, la mémoire, mais aussi la sexualité et la maladie mentale. Ratner développe également une théorie de psychologie macroculturelle qui met l'accent sur la dimension politique.

Il est intéressant de citer également les travaux de Nisbett, un auteur très productif au cours des dernières années. On mentionnera tout particulièrement la revue de littérature publiée dans la troisième édition du Handbook of experimental psychology (Nisbett et Norenzayan, 2002), et un livre consacré aux différences entre les manières de penser asiatique et occidentale (Nisbett, 2003). En conclusion de leur chapitre, Nisbett et Norenzayan soulignent que la pensée humaine se développe dans un contexte culturel et que les processus culturels affectent le fonctionnement cognitif. Ils montrent aussi la complémentarité de la psychologie et de l'anthropologie et remettent en question l'indépendance supposée des processus culturels et cognitifs. Ils expriment enfin l'espoir que l'examen interculturel de la cognition aidera à découvrir de véritables invariants cognitifs entre les cultures.

Parmi les contenus cognitifs probablement universels, ces auteurs mentionnent le fait que les bébés naissent en étant préparés à développer des modèles particuliers du monde, incluant des théories de la mécanique et une théorie de l'esprit. Ces contenus contraignent la diversité de la pensée humaine et des cultures possibles. En revanche ils pensent que les processus cognitifs sont moins universels que ce que les auteurs ont affirmé par le passé. De plus les pratiques culturelles et les processus cognitifs se constituent conjointement. Des pratiques culturelles favorisent et soutiennent certains types de processus cognitifs, qui ensuite perpétuent les pratiques culturelles.

Dans son ouvrage de 2003, Nisbett compare plus directement les manières de penser qui caractérisent les peuples du Sud-Est asiatique et celles des Occidentaux. II souligne qu'il peut y avoir des différences culturelles dans l'accessibilité cognitive aux processus de pensée. Ainsi les Asiatiques ont plus tendance que les Occidentaux à organiser le monde en termes de similarités et de relations, à expliquer les événements en fonction de la situation et à se baser sur des raisonnements reposant sur des connaissances. Les Occidentaux pour leur part ont plus tendance à organiser le monde en termes de catégories basées sur des règles, à expliquer les événements en termes de dispositions, et à se baser sur des raisonnements formels, décontextualisés.

\section{Convergences}

Les recherches comparatives qui ont marqué la première phase de la psychologie interculturelle ont diminué en importance au profit d'autres approches tenant largement compte de certaines critiques ou cherchant à prévenir les difficultés méthodologiques inhérentes aux études comparatives classiques. On peut y voir l'influence, directe ou indirecte, du courant relativiste. Le succès croissant à partir des années 1980 des idées inspirées de la théorie de Vygotsky a influencé d'une manière ou d'une autre les recherches sur le développement interculturel.

Nombre de chercheurs, après avoir pratiqué des études comparatives, ont évolué soit vers leur abandon pur et simple (Cole, 1995), soit vers des collaborations avec des scientifiques d'autres disciplines (Dasen, 1997), soit encore vers des études intra-culturelles. L'étude des savoirs quotidiens déjà mentionnée plus haut est un autre 
exemple de cette influence. C'est dans un esprit similaire que Dasen a ressenti le besoin de travailler en collaboration avec l'anthropologue Jürg Wassmann. La conjonction des préoccupations de chacune des disciplines a permis à ces chercheurs de définir une méthodologie originale qu'ils ont appliquée en Papouasie-Nouvelle-Guinée chez les Yupnos pour l'étude des classifications et à propos des concepts numériques (Wassmann et Dasen, 1994a et 1994b). Ces mêmes chercheurs ont ensuite étudié à Bali l'influence des systèmes d'orientation spatiale géocentrés sur la représentation de l'espace (Wassmann et Dasen, 1996). Dasen a poursuivi des recherches dans la même perspective en collaboration avec des chercheurs indiens (Mishra, Dasen et Niraula, 2003; Dasen, Mishra, Niraula et Wassmann, 2006; Dasen et Mishra, 2010).

Nos propres recherches sur le jeu d'awélé s'apparentent aux études sur les savoirs quotidiens. L'étude de ce jeu nous a paru d'autant plus intéressante que la littérature sur les jeux de plateau (board games) comporte de nombreuses études sur les jeux pratiqués en Occident (échecs surtout, mais aussi Othello, jeu de dames, Backgammon, etc.), mais que des lacunes béantes subsistent à propos des jeux provenant d'autres cultures, comme l'observaient déjà Hopkins et Wober en 1973. Les jeux offrent pourtant des situations particulièrement favorables pour entreprendre des études dans différentes cultures. Parmi les avantages qu'on peut mentionner figure le fait qu'il s'agit de situations précises, bien définies et auxquelles les sujets se prêtent volontiers. En outre, c'est un des domaines dans lesquels on peut s'attendre à trouver des experts dans la culture où le jeu étudié est pratiqué, sans qu'ils aient acquis cette expertise à travers des études scolaires ou des influences culturelles extérieures à leur propre groupe (Gobet, de Voogt et Retschitzki, 2004).

L'awélé appartient à la catégorie des jeux de semailles ou mancalas, dont plus d'une centaine de variantes ont été répertoriées, principalement en Afrique, mais également en Inde et dans les Caraïbes. C'est un jeu de stratégie dans la mesure où le hasard n'intervient pas: seuls les calculs des joueurs leur permettent de choisir le meilleur coup. Lors de plusieurs séjours en Côte d'Ivoire, nous avons donc étudié différents aspects de la pratique de ce jeu chez des enfants et des adultes (Retschitzki, 1990).

Ces recherches ont eu recours, comme celles de Dasen et Wassmann, à un ensemble de méthodes: simple observation des conduites, entretiens avec les joueurs, analyse détaillée des coups dans un grand nombre de parties enregistrées en vidéo, épreuves psychologiques ad hoc pour mesurer diverses compétences propres au jeu, etc. Elles ont permis de mettre en évidence plusieurs aspects concernant le rôle des facteurs susceptibles d'expliquer la supériorité des bons joueurs, la manière dont les enfants maîtrisent progressivement les finesses des stratégies propres à ce jeu, les processus à l'œuvre lors de la pratique de ce jeu, ainsi que certaines caractéristiques des stratégies des joueurs adultes.

L'analyse du dernier aspect s'est révélée plus délicate, faute d'une connaissance suffisante de certaines finesses du jeu de la part des chercheurs à l'époque de l'étude. Les analyses détaillées des parties, les entretiens avec certains des meilleurs joueurs de Côte d'Ivoire, les parties opposant de bons joueurs citadins aux meilleurs joueurs du village baoulé de Kpouébo et une partie didactique entre l'auteur (assisté de quelques bons joueurs) et le champion national ont toutefois permis de dégager certaines pistes et d'indiquer en quoi les experts adultes sont supérieurs aux meilleurs joueurs de 15 ans. Nous avons cherché à montrer que les conduites de ces meilleurs joueurs adultes (même illettrés) supposaient la présence de capacités cognitives équivalentes aux opérations formelles décrites par Piaget (Retschitzki, 1989). Actuellement, ce sont les caractéristiques des experts de ce jeu qui sont plus particulièrement étudiées grâce à des techniques faisant appel à des technologies plus récentes (Retschitzki, 2000). Une première expérience a permis de préciser le rôle de la mémoire de travail dans la représentation des situations de jeu (Retschitzki, Gygax et Richardson, article soumis).

\section{Conclusion}

Au terme de ce survol de quelques recherches interculturelles, il me semble qu'on peut affirmer que la culture est un facteur indéniable du développement cognitif. L'évolution des paradigmes de recherche a été marquée par un rapprochement des méthodes psychologiques et anthropologiques qu'on peut en partie attribuer aux critiques provenant du courant de la psychologie culturelle inspiré par l'approche historico-culturelle de Vygotsky. Du fait 
des limitations volontaires que ce courant s'est imposé en s'interdisant la comparaison considérée comme n'étant pas pertinente, on ne peut guère recenser les apports des travaux qui en sont issus.

L'une des convergences à souligner est que, pour ces auteurs aussi, la culture est un facteur déterminant du développement cognitif. D'ailleurs, on peut observer que nombre de concepts invoqués par les tenants de la psychologie culturelle semblent séduisants (étayage, zone proximale de développement, participation périphérique légitime, etc.), mais que leur formulation reste souvent trop floue pour donner lieu à des vérifications empiriques convaincantes.

Les travaux les plus récents, qu'il s'agisse des travaux récents de Greenfield (2004) sur le tissage, de nos propres travaux sur le jeu d'awélé, des travaux de Wassmann et Dasen sur les notions logico-mathématiques ou spatiales (Wassmann et Dasen, 1994a, 1994b et 1996) ou des travaux de Nisbett, qui se sont affranchis de l'interdit de la comparaison (Nisbett, 2003; Nisbett et Norenzayan, 2002), ouvrent de nouvelles perspectives de recherches qui permettront sans doute de mieux décrire les effets de la culture comme facteur du développement cognitif.

\section{Références bibliographiques}

Anderson, J., Greeno, J., Reder, L. et Simon, H. (2000). Perspectives on learning, thinking, and activity. Educational Researcher, 29(4), 11-13.

Anderson, J., Reder, L. et Simon, H. (1996). Situated learning and education. Educational Researcher, 25(4), 5-11.

Berry, J. (1976). Human ecology and cognitive style. New York : Sage/Halsted/Wiley.

Berry, J., Poortinga, Y., Segall, M. et Dasen, P. (2002). Cross-cultural psychology. Research and applications (2 éd.). Cambridge : Cambridge University Press.

Boesch, E. (1995). L'action symbolique. Fondements de psychologie culturelle. Paris : L'Harmattan.

Bril, B. et Lehalle, H. (1988). Le développement psychologique est-il universel? Paris : Presses Universitaires de France.

Bronfenbrenner, U. (1979). The ecology of human development: experiments by nature and design. Cambridge, Mass. : Harvard University Press.

Brown, J. et Burton, R. (1978). Diagnostic models for procedural bugs in basic mathematical skills. Cognitive Science, 2(2), 155-192.

Brown, J., Collins, A. et Duguid, P. (1989). Situated cognition and the culture of learning. Educational Researcher, $18(1), 32-42$.

Bruner, J. (1990). Acts of meaning. Cambridge, Mass. : Harvard University Press.

Bruner, J. (1991). Car la culture donne forme à l'esprit. De la révolution cognitive à la psychologie culturelle. Paris : Eshel.

Bruner, J. (1996). The culture of education. Cambridge, Mass. : Harvard University Press.

Bruner, J., Goodnow, J. et Austin, G. (1956). A study of thinking. New York : Wiley.

Cole, M. (1992). Culture in development. Dans M. Bornstein et M. Lamb (dir.), Developmental psychology. An advanced textbook (p. 731-789). Hillsdale : Erlbaum.

Cole, M. (1995). From cross-cultural to cultural psychology. Swiss Journal of Psychology, 54(4), 262-276.

Cole, M. (1996). Cultural psychology. A once and future discipline. Cambridge, Mass. : Harvard University Press.

Collins, A., Brown, J. et Newman, S. (1989). Cognitive apprenticeship: Teaching the crafts of reading, writing, and mathematics. Dans L. Resnick (dir.), Knowing, learning and instruction. Essays in honor of Robert Glaser (p. 453-494). Hillsdale : Erlbaum. 
Collins, A. et Loftus, E. (1975). A spreading-activation theory of semantic processing. Psychological Review, 82, 407428.

Collins, A. et Quillian, M. (1969). Retrieval time from semantic memory. Journal of Verbal Learning and Verbal Behavior, 8(2), 240-247.

Dasen, P. (1975). Concrete operational development in three cultures. Journal of Cross-Cultural Psychology, 6, 156172.

Dasen, P. (1997). Piaget entre relativisme et universalité. Dans C. Meljac, R. Voyazopoulos et Y. Hatwell (dir.), Piaget après Piaget. Évolution des modèles, richesse des pratiques (p. 135-153). Grenoble : La Pensée Sauvage.

Dasen, P. (2003). Theoretical frameworks in cross-cultural developmental psychology: An attempt at integration. Dans T. Saraswathi (dir.), Cross-cultural perspectives in human development (p. 128-165). New Delhi/Thousand Oaks, CA : Sage.

Dasen, P. (2004). Education informelle et processus d'apprentissage. Dans A. Akkari et P. Dasen (dir.), Pédagogies et pédagogues du Sud (p. 19-47). Paris : L'Harmattan.

Dasen, P. et Bossel-Lagos, M. (1989). L'étude interculturelle des savoirs quotidiens : revue de la littérature. Dans J. Retschitzki, M. Bossel-Lagos et P. Dasen (dir.), La recherche interculturelle (vol. 2, p. 98-114). Paris : L'Harmattan.

Dasen, P., Inhelder, B., Lavallée, M. et Retschitzki, J. (1978). Naissance de l'intelligence chez l'enfant baoulé de Côte d'Ivoire. Berne : Huber.

Dasen, P. et Mishra, R. (2010). Development of geocentric spatial language and cognition. Cambridge : Cambridge University Press.

Dasen, P., Mishra, R., Niraula, S. et Wassmann, J. (2006). Développement du langage et de la cognition spatiale géocentrique. Enfance, 58, 146-158.

Gobet, F., de Voogt, A. et Retschitzki, J. (2004). Moves in Mind: The Psychology of Board Games. Hove, UK : Psychology Press.

Greenfield, P. (1976). Cross-cultural research and Piagetian theory: Paradox and progress. Dans K. Riegel et J. Meacham (dir.), The developing individual in a changing world (p. 322-333). The Hague : Mouton.

Greenfield, P. (2004). Weaving generations together: Evolving creativity in the Maya of Chiapas. Santa Fe, NM : School of American Research Press.

Greenfield, P. et Lave, J. (1978). Aspects cognitifs de l'éducation non scolaire. Recherche, Pédagogie et Culture, 8, 16-35.

Hopkins, B. et Wober, M. (1973). Games and sports: Missing items in cross-cultural psychology. International Journal of Psychology, 8(1), 5-14.

Jahoda, G. (1980). Theoretical and systematic approaches in cross-cultural psychology. Dans $\mathrm{H}$. Triandis et W. Lambert (dir.), Hanbook of cross-cultural psychology. Perspectives (vol. 1, p. 69-142). Boston : Allyn \& Bacon.

Langley-Danysz, P. (1979). Le handicap du berceau. Sciences et Avenir, 392, 83-87.

Lave, J. (1988). Cognition in practice. Boston : Cambridge University Press.

Mishra, R., Dasen, P. et Niraula, S. (2003). Ecology, language, and performance on spatial cognitive tasks, International Journal of Psychology, 38(6), 366-383.

Nisbett, R. (2003). The geography of thought : how Asians and Westerners think differently ... and why. New York : Free Press.

Nisbett, R. et Norenzayan, A. (2002). Culture and cognition. Dans H. Pashler et D. Medin (dir.), Steven's handbook of experimental psychology. Memory and cognitive processes ( $3^{\mathrm{e}}$ éd., vol. 2, p. 561-597). Hoboken, NJ: Wiley. 
Nunes, T., Schliemann, A. et Carraher, D. (1993). Street mathematics and school mathematics. Cambridge : Cambridge University Press.

Ratner, C. (2002). Cultural psychology: Theory and method. New York : Plenum Press.

Ratner, C. (2006). Cultural psychology: A perspective on psychological functioning and social reform. Mahwah, NJ : Erlbaum.

Resnick, L., Levine, J. et Teasley, D. (1991). Perspectives on socially shared cognition. Washington : APA.

Retschitzki, J. (1989). Evidence of formal thinking in baoule awele players. Dans D. Keats, D. Munro et L. Mann (dir.), Heterogeneity in cross-cultural psychology (p. 234-243). Amsterdam : Swets \& Zeitlinger.

Retschitzki, J. (1990). Stratégies des joueurs d'awélé. Paris : L'Harmattan.

Retschitzki, J. (2000). Strategies of expert awele players. Dans N. Neuwahl (dir.), Proceedings of the International Colloquium "Board Games in Academia III" (p. 84-94). Florence.

Retschitzki, J., Gygax, P. et Richardson, R. (soumis). Warri experts and working memory.

Rogoff, B. (1990). Apprenticeship in thinking. Cognitive development in social context. Oxford : Oxford University Press.

Rogoff, B. (2003). The cultural nature of human development. Oxford : Oxford University Press.

Salomon, G. (1993). Distributed cognitions. Psychological and educational considerations. Cambridge : Cambridge University Press.

Scribner, S. (1969). The cognitive consequences of literacy (manuscrit non publié). City University of New York.

Segall, M., Dasen, P., Berry, J. et Poortinga, Y. (1999). Human behavior in global perspective. An introduction to cross-cultural psychology ( ${ }^{\mathrm{e}}$ éd.). New York : Allyn \& Bacon.

Shweder, R. (1990). Cultural psychology-what is it? Dans J. Stigler, R. Shweder et G. Herdt (dir.), Cultural psychology: Essays on comprative human development (p. 1-43). Cambridge : Cambridge University Press.

Super, C. et Harkness, S. (1986). The developmental niche: a conceptualization at the interface of child and culture. International Journal of Behavioral Development, 9(4), 545-570.

Tanon, F. (1994). A cultural view on planning: The case of weaving in Ivory Coast. Tilburg : Tilburg University Press.

Tapé, G. (1994). L'intelligence en Afrique. Une étude du raisonnement expérimental. Paris : L'Harmattan.

Troadec, B. (2007). Psychologie culturelle. Le développement cognitif est-il culturel ? Paris : Belin.

Wassmann, J. et Dasen, P. (1994a). "Hot and cold": Classification and sorting among the Yupno of Papua New Guinea. International Journal of Psychology, 29, 19-38.

Wassmann, J. et Dasen, P. (1994b). Yupno number system and counting. Journal of Cross-cultural Psychology, 25(1), 78-94.

Wassmann, J. et Dasen, P. (1996). Comment ne pas perdre le Nord à Bali. Processus cognitifs - Une combinaison de méthodes ethnographiques et psychologiques. Bulletin de l'Académie Suisse des Sciences Humaines et Sociales, 1, 17-26.

Wundt, W. (1912). Elemente der Völkerpsychologie : Grundlinien einer psychologischen Entwicklungsgeschichte der Menschheit. Leipzig : A. Kröner. 